\title{
13-Defects, Microstructures and Textures
}

- minute misorientations are present between the different parts of a given cell,

- the contrasts of the regions connecting the facets at the tip of the cells and in the grooves between them are different. This phenomenon is not related to the sign of a possible local curvature of the net planes in these regions. Up to now it is not elucidated and electron microscopic observations are needed which could give further experimental information about the general question of the transition between plane and rough interface at the facet edge.

PS-13.02.16 X-RAY SECTION TOPOGRAPHY STUDY OF BRAZIL TWIN BOUNDARIES IN NATURAL AMEFHYST. BY Z. Baran', Instituto de Fisica, Universidade Federal da Bahia, Salvador, Ba., Brazil and B. Capelle, Laboratoire de Minéralogie-Cristallographie, Université $P$. et $M$. Curie, 75252 Paris, France.

A natural amethyst crystal twinned according to the Brazil twin law was investigated by means of $X$-ray section topography in order to explain the unexpected contrast between altemating dextrogyre and levogyre lamellae formed at the natural rhomboedral face (0111) of this Brazilian amethyst crystal. It was also studied by transmission projection topography, recorded with $\mathrm{Mo} \mathrm{K} \alpha$ radiation and $\mu \mathrm{t}=0,3$ (Z. Baran et al., 1990, Acta Cryst., A46, p. C-430). Under such a condition of relatively low absorption, the contribution of anomalous dispersion to the image contrast should be rather small. It was found, however, that the Brazil twinning, which can also be considered as an inversion twinning, can be observed by means of X-ray topography.

The Brazil twin consists of dextrogyre and levogyre lamellae in parallel orientation with $(11 \overline{2} 0)$ or $\{10 \overline{1} 1\}$ as composition plane. This is really a parailel growth (Lu Taijing and I. Sunagawa, 1990, J. Cryst. Growth, 99, 1232). In a previous paper (Z. Baran et al., 1987. Phys. Stat. Sol. (a), 101, 9 ), a difference in the lattice spacing in the direction perpendicular to the rhomboedral growth face was observed by X-ray double-crystal topography between the twin lamellae and the twin boundary layer, and shown to be equal to $\Delta d / d=-2 \cdot 10^{-5}$. This fact agrees well with a probable model of accommodation layer which is formed between the adjacent twin lamellae. As a consequence, lattice deformations can be also expected along the twin boundaries. To investigate the nature of contrast images in our projection topographs, a series of section topographs was taken at different positions. These section topographs show that the interface or the boundary is detectable by X-ray topography. The observed very complicated contrast image is not similar to that occuring in the Brazil twin boundary in natural quartz, as it was observed by $Y$ ang et al., (1986, Phys. Stat Sol. (a) 97, 411), which is characterized by an hour-glassshaped system of fringes. Thus, it is presumed, the source of the image contrast of the twin boundary is to be associated with strain. This corresponds to the last item of the classification of the surface defects separating two perfect or nearly perfect regions of the crystal (A.Authier, 1977, in Crystal Growth and Materials, Chap. II.3, ed. by E. Kaldis and H.J. Scheel, North Holland Publ., p.530). The enhanced black-andwhite contrast observed on the $\mathrm{X}$-rays projection photographs along the Brazil boundaries shows that a strong strain field exists within the twin lamellae, reflecting the same structure factor modulus (i.e. no domain contrast). As indicated by our section topographs, the observed contrasts do not present a dynamical but rather a kinematical character due to strain concentrations at the boundary. An attempt is made to explain the nature of those boundaries by means of a detailed discussion of the recorded topographs.

Support from Laboratoire de Minéralogie-Cristallographie, Université P. et $\mathrm{M}$. Curie and $\mathrm{CNPq}$ (Brazil) is acknowleged.

PS-13.02.7 VIBRATION MODES STUDIES USING SYNCHROTRON X-RAY TOPOGRAPHY. B. Capelle, I Détaint, J. Schwartzel, A. Zarka*, Y. Zheng. Laboratoire de MineralogieCristallographie, Universités P. \& M. Curie (Paris VI) et Paris VII, CNRS 09,4 place Jussieu, 75252 Paris Cedex 05; France. Centre National d'Etudes des Télécommunications,PAB/B AG/MCT, 92220 Bagneux, France.

$\mathrm{X}$-ray topography bas been extensively used to assess the quality of crystals, in particular for quartz, and it is now a usual technique to characterize vibration modes. More recently, the availability of synchrotron radiation bas brought new possibilities to this technique : the high flux of $\mathrm{X}$-ray radiation pernits to observe simultaneously several diffraction patterns with different diffraction vectors $g$ and to analyse the spatial structure of vibration modes; the synchrotron pulsed $\mathrm{X}$-ray beam can be used to excite $X$-ray synchronized vibrations in resonators and it allows to reveal the time structure of vibration modes. Synchrotron $X$-ray topography appears thus to be a well adapted method of analysing vibration mode sbapes. Synchrotron $X$-ray topography permits also to verify experimentally theoretical models and to get informations not yet considered experimentally particular cases, complex boundary conditions and coupling piez theoretically. For may lead new components in the displacement fields Experimentectric constants DCI siorage ring (France) have displacement fields. Experiments at the LUREquartz rese ring (France) have been carried out to characterize mode shapes in quartz resonators, but also in berlinite and lithium tantalate, and in connection with the presence of different crystal defects. Interesting cases showing stationary trapped and coupled components, were evidenced by classical X-ray topographs in quartz AT plano-convex resonators. These stroboscopic topographs provide interesting experimental data for the investigation of non-linear effects in quartz. In a similar way, quartz of other cuts (BT and SC), berlinite and lithium tantalate have been investigated and instructive informations were obtained concersing mode lateral anisotropy, coupled components eic. Other important cases studied by synchrotron $X$-ray topography concem crysil Oef important cases studied by growth bands) which affect mode shapes in a most complicated way acations and conditions such as stress and tore conchest that synchrouron $X$-ray topography provides useful experimental data concerning diverse aspects of mode shapes in resonators. Different factors affecting mode shapes such as coupling mechanisms, crystal defects and non-linear effects can be finely analysed by this technique.

PS-13.02.18 IMAGE TREATMENT OF SYNCHROTRON TOPOGRAPHS. By Y. Epelboin*, M. Pilard, A. Soyer, Lab. Minéralogie-Cristallographie, Universités P.M. Curie et Paris VII U.A. 09 CNRS, 75252 Paris Cedex 05, France, e-mail: epelboin@lmcp.jussieu.fr

The development of synchrotron facilities means that the processing and analysis of images recorded either on films or by means of TV cameras must be enhanced to extract the maximum number of features from a single image. The experiments are too costly and the time of experience too short to loose part of a possible information. Different mathematical treatments may be used for the same image if one wants to study different features.

In preliminary experiments for the ESRF, conducted at LURE/DCI, we have recorded digitized images directly from a X-Ray camera or later from films using an ordinary video camera. We have studied the influence of the digitization process on the quality of the images and we have investigated various techniques of image treatment such as Fourier filtering or maximum entropy. Wavelets are under investigation. 